\title{
EDITORIAL
}

\section{Healthcare Transformation}

Jonathan Linkous

Healthcare transformation has become a widely used phrase. Searching the phrase on Google yields a half million results. Spurred by rising costs and increased needs, the healthcare industry faces pressure to deliver more services to more people, at higher level of quality, and with greater efficiency. The coinciding transformation of technology and telecommunications as well as new payment approaches has resulted in opportunities for change.

But such opportunities often fail in healthcare. Prolonged efforts are needed to achieve success. All too often efforts to transform mistake means for ends. Hospitals, health systems, and even private practices launch quality improvement initiatives that are only activities or processes with little impact or improvement. As the saying goes, most improvement efforts have as much impact on company performance as a rain dance has on the weather. ${ }^{1}$

Nowhere is this mismatch clearer than the billions of dollars spent on new health applications and technology. Products and applications are too often a solution-lookingfor-a-problem. This is especially distressing because technology can, when used appropriately and embedded within other transformations, result in real, significant, and lasting improvements in access, quality, and efficiencies. A glaring example of this problem is the push for collecting more and more patient data with little effort to use it, only resulting in higher costs, frustrated providers, and modest change in patient outcomes. Amassing data alone is not a solution.

There are bright spots. For 25 years, I worked to bring about widespread adoption of telemedicine. That technology continues to make significant improvements in gaining access to care, bringing health services to those without. Today, almost 20 million patients receive health services at a distance. For years, telemedicine also strived to be considered only as good as face-to-face healthcare. Teamed with artificial intelligence (AI), robotics, sensors, and other technologies, significant improvements can be realized in health outcomes, increased productivity, and expanded access to care.

Real transformation is the challenge Healthcare Transformation hopes to help achieve. The journal brings together a unique mixture of original research, position articles, interviews of key leaders, profiles of successful initiatives, and solicited editorials. Instead of rain dances, it sets out to demonstrate how combinations of these 21 st century technologies are revolutionizing the way worldwide healthcare is delivered.

The priority of the journal is to both inform and assist a broad audience that includes government, health, and technology decision-makers; influential innovators within medicine and hi-tech industries; hospital and pharmaceutical executives; healthcare academicians and educators concerned with healthcare; and advanced technology applications.

Topics of interest include:

- Clinical practices incorporating AI, robotics, telemedicine, sensors, and other advanced technologies.

- Public policy and regulation affecting the use of advanced technology in health delivery.

- Patient acceptance.

- Operational and business practices to successfully integrate advanced technologies into healthcare offices and systems.

- Services using an integrated approach of multiple technologies that are both interoperable and work harmoniously, which result in significant improvements in healthcare quality, productivity, and/or access.

- Emerging technology-based applications for consumer health.

- Advances in robotic applications in health delivery, pharmaceutical, and related areas.

We hope that you will find these contributions will bring insights and prompt efforts to achieve real sustained improvements in the delivery of healthcare. We welcome your contributions and comments.

\section{Reference}

1. Schaffer RH, Thomson HA. Successful change programs begin with results. Harvard Bus Rev. 1992. https://hbr.org/1992/01/successful-changeprograms-begin-with-results. (Last accessed on November 6, 2019). 